\title{
Cárie precoce e severa na infância: a exodontia precoce pode prejudicar a qualidade de vida de crianças acometidas?
}

\author{
Early and severe caries in childhood: early extraction can impair the quality of life of \\ affected children?
}

Caries temprana de la infancia severa ¿puede la exodoncia precoz de un diente afectar la calidad de vida de los niños afectados?

Thayná Amiche ${ }^{1}$, Fernanda de Oliveira Marcico ${ }^{1}$, Gabriele Rocha ${ }^{1}$, Diego de Andrade Teixeira $^{1 *}$, Luciana Alves Herdy da Silva ${ }^{1}$, Flávia Cariús Tesch Ferreira Alves ${ }^{1}$, José Massao Miasato ${ }^{1}$, Leila Chevitarese ${ }^{1}$.

\section{RESUMO}

Objetivo: Verificar se a exodontia precoce pode prejudicar a qualidade de vida de crianças de 6 a 8 anos de idade, acometidas por cárie precoce e severa na infância. Métodos: Três escolas participaram da pesquisa, com crianças de seis a oito anos. Um questionário foi aplicado para avaliar rotinas e o histórico de saúde da criança. Também foi elaborado um prontuário clínico individual, contendo os índices ceo-d e CPO-D, para avaliar a experiência de cárie em dentes decíduos e permanentes, respectivamente. O estudo foi aprovado por Comitê de Ética em Pesquisa. Resultados: Foi avaliado um total de 141 voluntários. A maior prevalência de cárie dentária foi encontrada em 76,56\% dos voluntários da escola pública, seguida $72,73 \%$ nos da escola particular (Qui², $p=0,744)$. Houve dificuldade de fala ou interação social em 15,62\% e 7,79\% nas crianças da escola pública e da escola particular, respectivamente. Conclusão: Houve a presença de cárie de estabelecimento precoce nas crianças do estudo e tal presença pode interferir na qualidade de vida dos voluntários.

Palavras-chave: Cárie dentária, Qualidade de vida, Odontopediatria.

\begin{abstract}
Objective: To verify if an early tooth extraction can harm children's quality of life from 6 to 8 years old, affected by early and severe caries in childhood. Methods: Three schools participated in the survey, with children aged six to eight years. A questionnaire was designed to assess routines and the child's health history. Researchers also prepared an individual clinical record, and the ceo-d and CPO-D indexes were used to evaluate caries' experience in primary and permanent teeth, respectively. The study was approved by the Research Ethics Committee. Results: A total of 141 volunteers were evaluated. The highest prevalence of dental caries was $76.56 \%$ of volunteers from the public school, then $72.73 \%$ in those from the private school (Qui ${ }^{2}, p=0.744$ ). There is difficulty in speaking or social interaction in $15.62 \%$ and $7.79 \%$ of children from public and private schools, respectively. Conclusion: There was a presence of caries of early establishment in the children of the study, and such presence can interfere in the volunteers' quality of life.
\end{abstract}

Keywords: Dental caries, Quality of life, Pediatric dentistry.

\section{RESUMEN}

Objetivo: Verificar si la extracción dentaria temprana puede afectar la calidad de vida de los niños de 6 a 8 años, quienes se ven afectados por caries temprana y severa en la infancia. Métodos: Tres escuelas participaron en la encuesta, con niños de seis a ocho años. Se aplicó un cuestionario para evaluar las rutinas y el historial de salud del niño. También se elaboró una historia clínica individual, que contiene los índices ceo-d y CPO-D, para evaluar la experiencia de caries, en dientes temporales y permanentes, respectivamente. El estudio fue aprobado por el Comité de Ética en Investigación. Resultados: Se evaluaron un total de 141 voluntarios. La mayor prevalencia de caries dental se encontró en el 76,56\% de los voluntarios de la escuela pública, seguido por el $72,73 \%$ en los de la escuela privada $\left(Q u i^{2}, p=0,744\right)$. Hubo dificultad en el habla o la interacción social en $15,62 \%$ y $7,79 \%$ en niños de escuelas públicas y privadas, respectivamente. Conclusión: Existió la presencia de caries de establecimiento temprano en los niños del estudio y tal presencia puede interferir en la calidad de vida de los voluntarios.

Palabras clave: Caries dental, Calidad de vida, Odontopediatría.

1 Universidade do Grande Rio (UNIGRANRIO), Duque de Caxias - RJ.

*E-mail: diegodeandradeodontologia@gmail.com

SUBMETIDO EM: 5/2021

ACEITO EM: 5/2021

PUBLICADO EM: 5/2021 


\section{INTRODUÇÃO}

A cárie dentária representa um grande problema de saúde pública e fatores socioeconômicos, níveis educacionais e estilo de vida podem estar relacionados com o seu aparecimento. A família tem influência direta na vida da criança e dos seus hábitos alimentares, por isso é de grande importância que os familiares aprendam e saibam reconhecer os sinais e sintomas relacionados com o aparecimento da cárie dentária, para modificar os fatores de risco causadores de doenças nas crianças, a fim de participarem do controle em nível de saúde pública (BUSATO AL, et al., 2014).

A falta de conhecimento da população sobre o controle dos fatores de risco relacionados com a cárie precoce e as dificuldades de acesso ao Sistema Único de Saúde (SUS) da população carente, podem se tornar a principais causas de perda precoce, resultando em grandes sequelas (MALTZ M, et al., 2016). As crianças brasileiras apresentam um dos mais altos índices de extrações dentárias prematuras, sem manutenção do espaço criado, o que pode ocasionar a redução do perímetro do arco e aumento da sobremordida entre outros problemas funcionais (OWEN DG, 1971).

É de suma importância que os profissionais da área façam o exame clínico e radiográfico de forma minuciosa para alcançar diagnóstico correto e que resulte em tratamento efetivo, com a participação do núcleo familiar possa gerar atitude preventiva no que diz respeito ao aparecimento da cárie precoce (BUSATO AL, et al., 2014). Pois a perda precoce de um dente decíduo pode acarretar diversos problemas entre eles, os mastigatórios, os de articulação, os de oclusão, os de fonação, os de estética, mudança na sequência de erupção e impactação dentária. Além disso, podem ocorrer impactos sociais na vida da criança, como o bullying (OWEN DG, 1971; THOMAZ EBA, et al., 2002).

O bullying pode ser conceituado como uma experiência comum na infância, acometendo crianças de diferentes condições socioeconômicas e culturais (COPELANDA WE, et al., 2014). No ambiente escolar, o bullying tem consequências no processo de socialização, ensino/aprendizagem, no que diz respeito à integridade física e psicológica dos alunos. Desta forma, é de suma importância identificar e intervir precocemente por parte dos professores, bem como um trabalho conjunto com profissionais de saúde, como institui o Programa Saúde na Escola, por exemplo (BRASIL, 2007).

O presente estudo teve por objetivo verificar se a exodontia precoce pode prejudicar a qualidade de vida de crianças de 6 a 8 anos de idade acometidas por cárie precoce e severa na infância.

\section{MÉTODOS}

Foi realizada uma pesquisa em três escolas (uma pública (EPU) e duas privadas (EPR)) em um Município da Baixada Fluminense situado no Estado do Rio de Janeiro em crianças que lá estudam, com idade compreendida entre seis e oito anos.

Os pais receberam o Termo de Consentimento Livre e Esclarecido (TCLE) e o questionário via agenda escolar. Só as crianças autorizadas pela assinatura do TCLE foram incluídas no estudo.

O questionário enviado para os pais, contendo perguntas abertas e fechadas, buscou avaliar rotinas e história pregressa da criança, relacionadas com: 1) frequência de ingestão de doces e de escovação dentária diárias; 2) se a escovação é supervisionada; 3) se usa creme dental fluoretado; 4) tempo decorrido da última visita ao dentista e sua razão; 5) se sente ou sentiu dor de dente, se já extraiu dente no dentista e qual a razão, se o dente era permanente ou decíduo, qual a condição do elemento dentário; 6) se sente dor ao mastigar; 7) se em caso de perda precoce do elemento decíduo, houve a colocação de mantenedor de espaço; 8) e se em caso de perda precoce de dente decíduo anterior, ainda com a ausência do seu sucessor permanente anterior, se a criança apresenta alguma dificuldade de fala, escrita ou interação social.

Uma ficha clínica individual para as crianças também foi elaborada e nela continha os índices ceo-d e CPO-D, utilizados para avaliar a experiência de cárie em dentes decíduos e permanentes, respectivamente e três perguntas que também estiveram no questionário enviado aos responsáveis (perguntas 6 e 8). A 
presença de biofilme dentário também foi registrada. As crianças receberam pastilha de fucsina básica a $2 \%$, e após mastigarem, espalharem sobre os dentes e lavarem a cavidade bucal para a remoção do excesso, observou-se a presença ou ausência de biofilme dentário. $O$ corante, também teve caráter didático, pois auxiliou na instrução mecânica da escovação.

Antes do início do exame as crianças tiveram seus dentes escovados com escova de dente e dentifrício fluoretado. O exame foi realizado com a criança de pé, de frente para o examinador. Uma lanterna foi utilizada para iluminar a cavidade bucal das crianças. O examinador contou com o auxílio de abaixador de língua para afastar os tecidos moles e gaze para secar os dentes. Os resultados foram anotados na ficha de exame clínico elaborada para este fim. No total foram 3 examinadoras, sendo 26 crianças examinadas por dia (período da manhã e tarde). As professoras foram avisadas previamente sobre os dias do exame e liberaram as crianças de $3 \mathrm{em} 3$ para serem avaliadas, que receberam orientação sobre a escovação dentária e uso do fio dental. Ao final, as crianças foram reconduzidas para a sala de aula, para que outras três pudessem passar pelo mesmo processo.

O presente estudo recebeu a aprovação do Comitê de Ética em Pesquisa da UNIGRANRIO (CAAE no 15374319.3.0000.5283).

\section{RESULTADOS}

No presente estudo foram selecionadas três escolas, sendo entregues 100 fichas para cada uma participante contendo o TCLE e um questionário para os responsáveis. Das 300 fichas entregues, 79,5\% (159 crianças) não deram resposta ao convite para participar da pesquisa. Fizeram parte da amostra do estudo 77 crianças das EPR e 64 da EPU, totalizando 141 crianças, sendo a maioria do sexo masculino (EPR: $51,94 \%$ (40); EPU: 53,12\% (34)).

No total de examinados, a maior prevalência de cárie dentária foi encontrada nos participantes da EPU $76,56 \%$ (49) seguido das EPR 72,73\% (56), no entanto essa diferença não foi significativa (Qui $\left.{ }^{2}, p=0,744\right)$. A maior prevalência de cárie dos escolares foi no lado direito das arcadas, sendo encontrada em 21 $(27,27 \%)$ participantes da EPR e em $18(28,12 \%)$ da EPU. Os alunos da rede pública apresentaram maior prevalência da doença, expressa por meio do CPO-d e seus componentes (Figura 1).

Figura 1 - Achados clínicos a partir do exame oral em crianças do estudo.

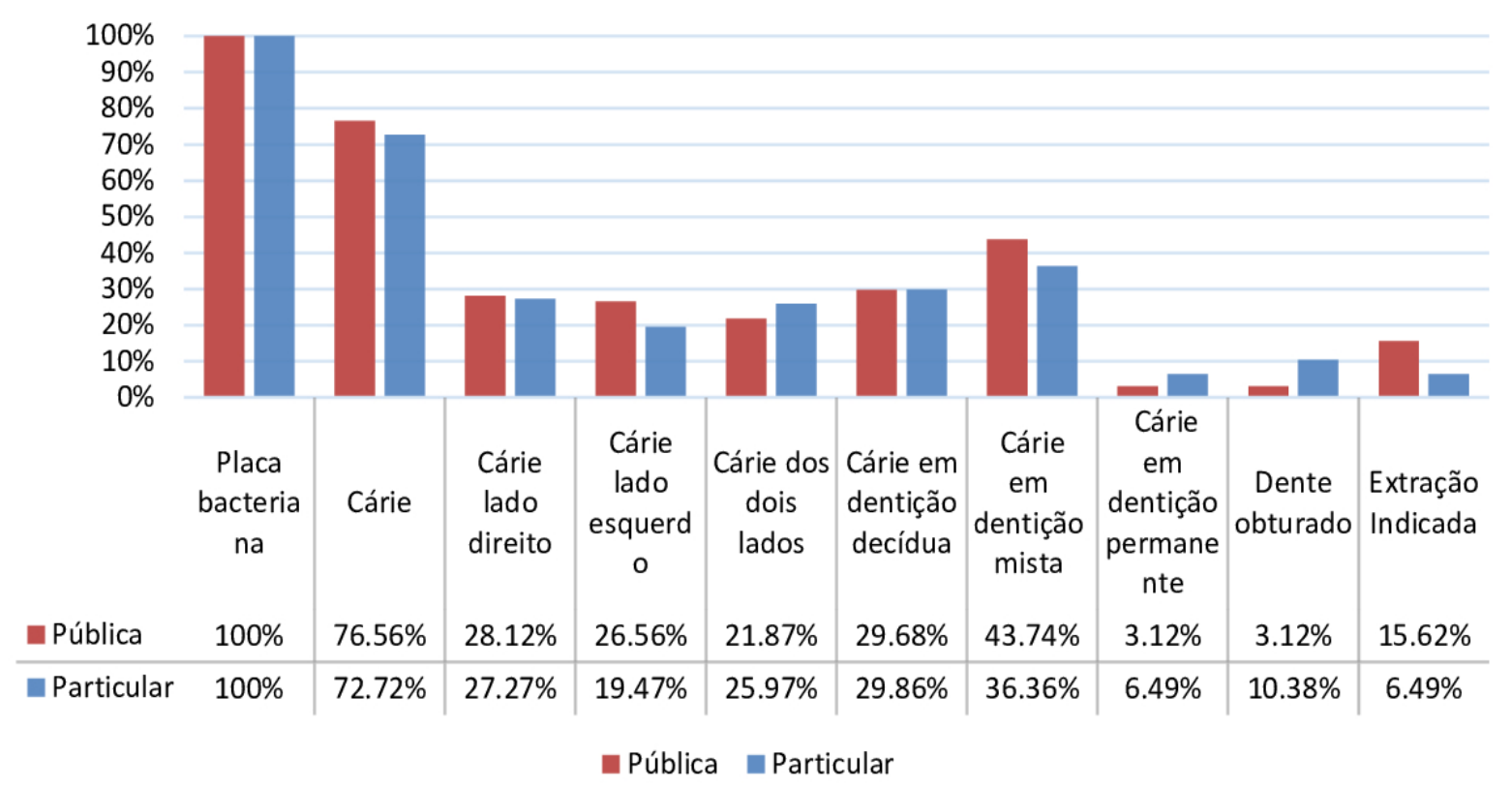

Fonte: Amiche T, et al., 2021.

O ceo-d encontrado na EPR e EPU (Figura 2) foi igual a 2,92 $\pm 2,81$ e 4,1 $\pm 3,42$, respectivamente, com diferença estatisticamente significante (Qui2, $p=0,015)$. O número total de dentes envolvidos com o índice 
ceo-d na EPR foi de 225. O número de dentes cariados foi de 202 (89,77\%), os com extração indicada foi de $10(4,44 \%)$ e, os obturados foi de 13 (5,77\%). Na EPU, o total de dentes envolvidos no índice ceo-d foi igual a 263. O número de dentes cariados foi de 241 (91,63\%), os com extração indicada foi de 20 (7,6\%) e, os obturados foi de $2(0,76 \%)$.

Figura 2 - Componentes do ceo-d das EPR e EPU.

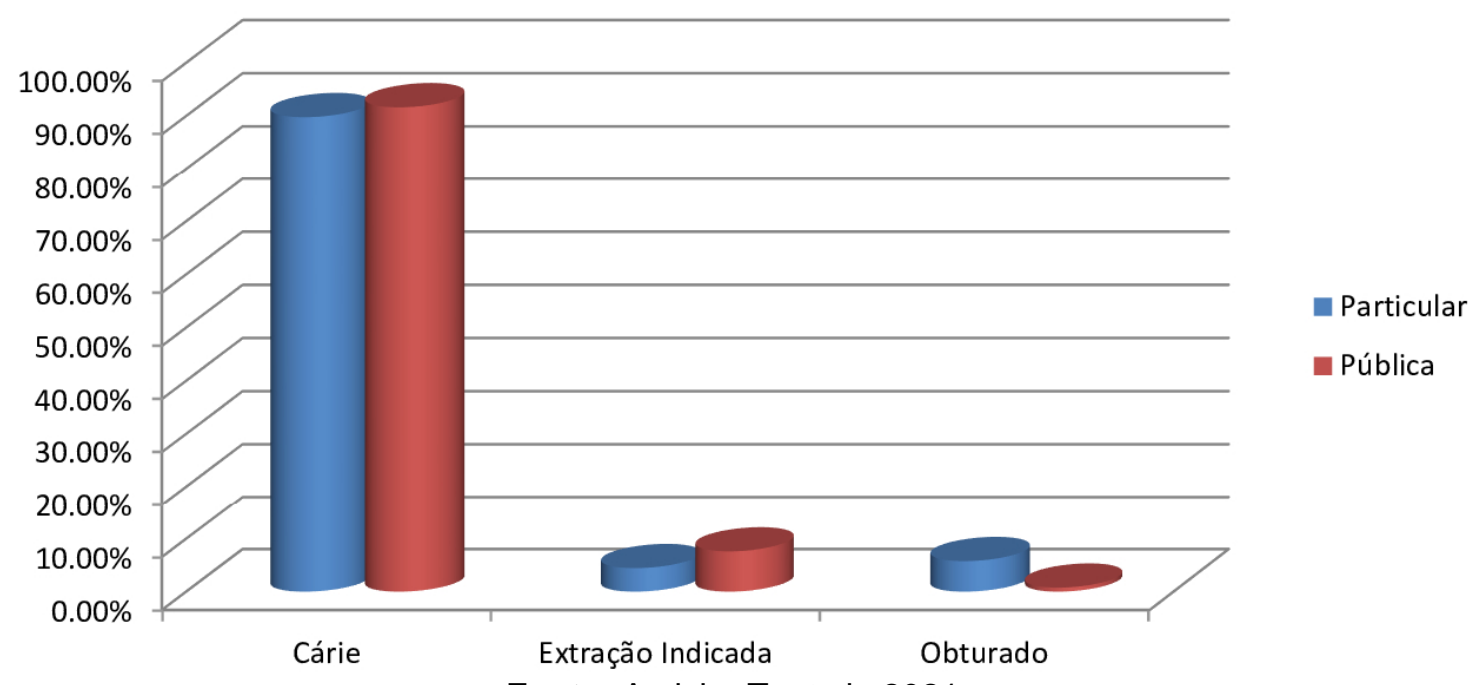

Fonte: Amiche T, et al., 2021.

O CPO-D encontrado na EPR e EPU foi igual a 1,01 $\pm 1,30$ e 1,28 $\pm 1,53$, respectivamente sem diferença estatisticamente significante (Qui2, $p=0,216)$. O número total de dentes envolvidos com o índice CPO-D na EPR foi de 78. O número de dentes cariados foi de 75 (96,15\%), e, o número de obturados foi de $3(4 \%)$. Na EPU o total de dentes envolvidos no índice CPO-D foi igual a 82 . Todos os dentes (100\%) estavam cariados. Em ambas as escolas, não foram encontrados o componente perdido, e na EPU não havia dente obturado (Figura 3).

Figura 3 - Componentes do CPO-D das EPR e EPU.

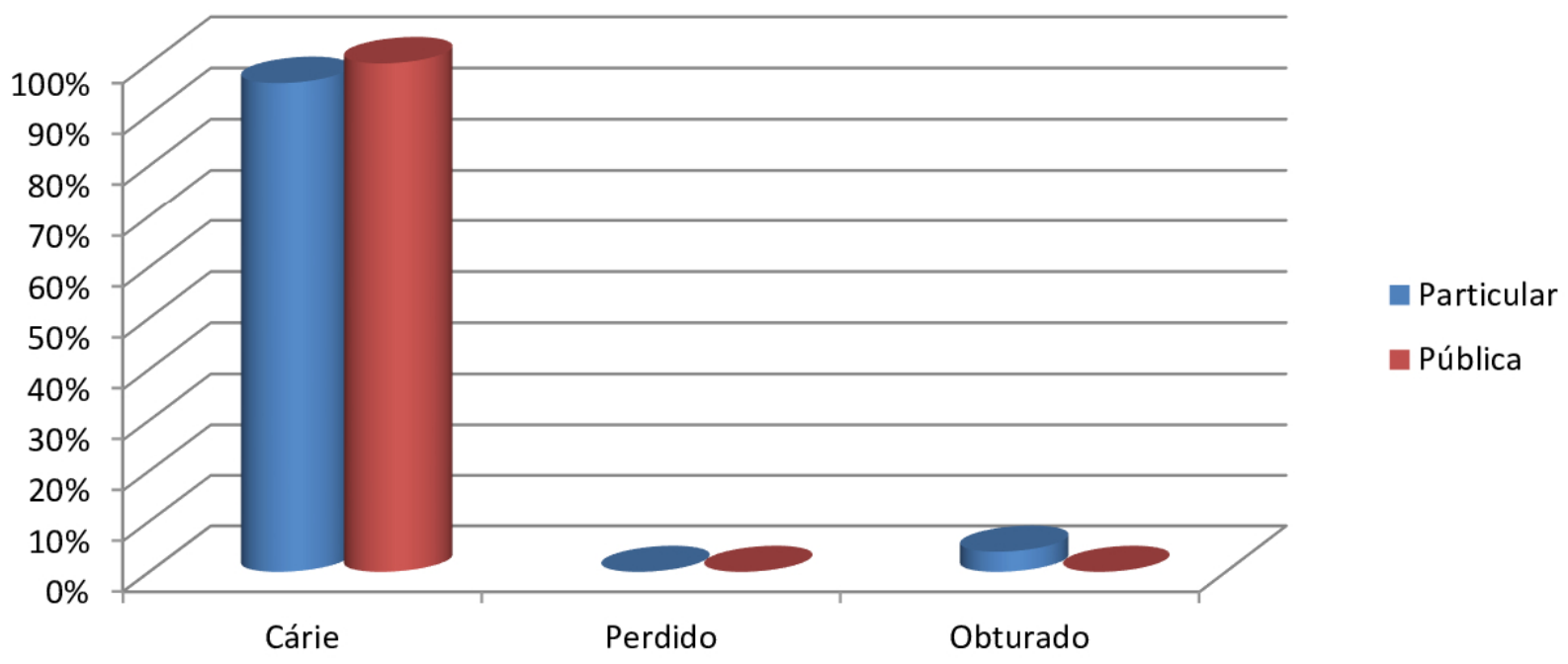

Fonte: Amiche T, et al., 2021.

Em relação ao questionário apresentado aos pais, foi observado que $67,18 \%$ (43) dos alunos da EPU nunca foram ao dentista, sendo que esta ida foi inferior em relação à EPR com 57,14\% (33); tendo como resposta clínica, a prevalência de cárie na pública ser mais elevada em relação à da privada. Observa-se também que 70,12\% (54) dos alunos da EPR são supervisionados durante a escovação, contra 65,62\% (42) da rede pública (Figura 3), sendo esta diferença não significativa (Qui; $p=0,697$ ). 
Foi perguntado aos pais a respeito do acesso ao dentista e o tempo decorrido da última consulta. Nas EPR, 54,7\% (29) crianças foram ao dentista nos últimos 12 meses, sendo que, 34,78\% (16) e 23,91\% (11) fizeram exames de rotina e limpeza, respectivamente. Quando perguntado se a criança sentia dor, $77,35 \%$ (41) dos responsáveis afirmaram que não. Na EPU, 42,18\% (27) crianças já foram ao dentista, destas, 48,14\% (13) nos últimos 12 meses. Os motivos apontados foram: 39,13\% (19) consulta de emergência, $30,43 \%$ (7) por tratamento de rotina. A ida ao dentista foi um dado significativo quando se comparam as EPR e EPU (Qui2; $p=0,007)$.

Todas as crianças do estudo apresentam idade compreendida entre 6 e 8 anos. Nestas crianças há a presença de todos os molares decíduos. No questionário enviado aos pais foi perguntado se houve perda dentária dos dentes decíduos anteriores e se havia a presença de cárie nos mesmos. Foi perguntado aos pais de crianças que tiveram caso de perda precoce de dente decíduo anterior, e ainda estavam com a ausência do seu sucessor permanente anterior, se a criança apresenta alguma dificuldade de fala, escrita ou interação social. Os resultados encontrados nas EPR mostraram que $41,55 \%$ (32) crianças já não apresentavam mais seus dentes decíduos anteriores e destas, $84,37 \%$ (27), responderam que estes apresentavam lesões de cárie e 78,12\% (25), não sentiam dor nos dentes que foram extraídos. Na EPU, $26,56 \%$ (17) de crianças se encontravam nessa situação e, 82,35\% (14) tiveram lesões de cárie e, $62,5 \%$ (10) apresentavam dor nesses elementos.

A Figura 4 evidencia o resultado das respostas dos responsáveis sobre a criança ter sentido dor. Eles demostraram que $45,31 \%$ e $25,9 \%$ das crianças, nas EPU e EPR, respectivamente sentiram dor e esta diferença foi significativa (Qui2; $p=0,026)$.

Também foram avaliadas as dificuldades de fala e de interação social das crianças que apresentaram ausência dos elementos dentários decíduos anteriores ausentes devido à extração precoce. Os resultados mostraram que $15,62 \%$ e $7,79 \%$ das crianças das EP e EPR, respectivamente, vivenciaram tais experiências, sem diferença estatística entre os grupos ( $Q u i 2 ; p=0,233$ ), no entanto prejudicou a qualidade de vida destas crianças.

Figura 4 - Respostas dos responsáveis ao questionário.

Dificuldade de fala ou interação social

Nunca foi ao dentista

Escovação supervisionada

Sente dor
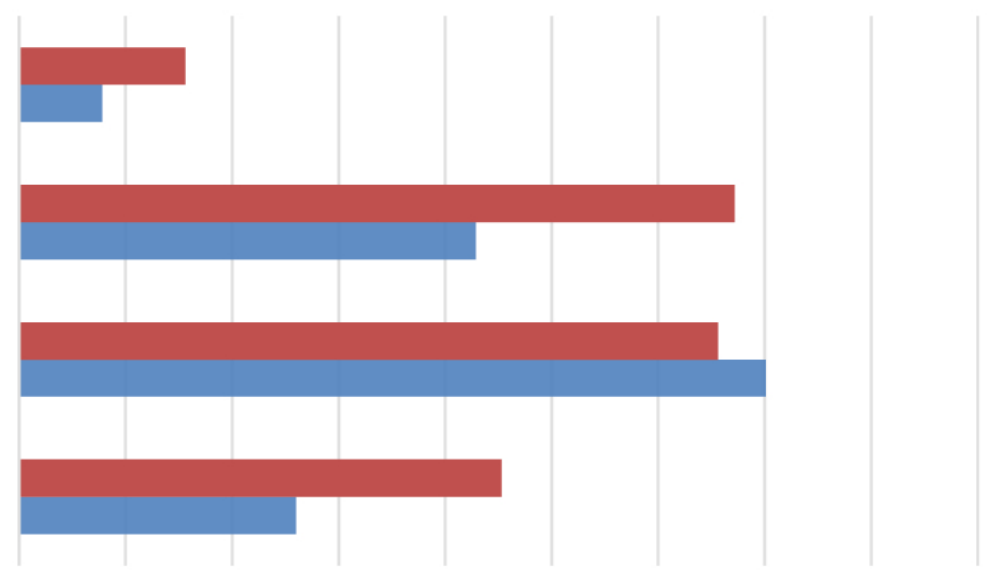

$0.00 \% \quad 10.00 \% \quad 20.00 \% 30.00 \%$ 40.00\% $50.00 \%$ 60.00\% $70.00 \%$ 80.00\% $90.00 \%$

\begin{tabular}{|l|c|c|c|c|}
\hline & Sente dor & Escovação supervisionada & Nunca foi ao dentista & $\begin{array}{c}\text { Dificuldade de fala ou } \\
\text { interação social }\end{array}$ \\
\hline E Pública & $45.31 \%$ & $65.62 \%$ & $67.18 \%$ & $15.62 \%$ \\
\hline - Privada & $25.97 \%$ & $70.12 \%$ & $42.85 \%$ & $7.79 \%$ \\
\hline
\end{tabular}

Fonte: Amiche T, et al., 2021.

Outro resultado a ser destacado é o fato de crianças nunca terem ido ao dentista, sentiram dor de dente, e não tiveram essa necessidade atendida. Na EPU 36,84\% (14) crianças de 38 que nunca foram ao dentista, sentiam dor. Nas EPR, das 42 crianças com a mesma situação 23,81\% (10) sentiam dor. 


\section{DISCUSSÃO}

O objetivo do presente estudo foi verificar se o estado de saúde bucal, expresso na qualidade dos elementos decíduos, no tocante à cárie dentária, interfere na qualidade de vida destes pacientes submetidos à perda precoce. A perda precoce derivada da cárie dentária apresenta um impacto negativo na qualidade de vida de saúde bucal e geral da criança e de seus familiares. O conceito de qualidade de vida relacionada à saúde bucal se relaciona ao impacto que a saúde ou a doença bucal tem no funcionamento diário, no bem-estar ou na qualidade de vida dos indivíduos. A cárie precoce pode interferir na alimentação, afetar o desenvolvimento da dentição permanente e até mesmo comprometer o crescimento e desenvolvimento da criança (MCDONALDS RE, et al., 1994).

De acordo com Matos NA (2002), 60\% das extrações precoces ocorrem em crianças até aos 8 anos de idade, aproximadamente. Já Bezerra ESM e Nogueira AJS (2012), constataram que a extração precoce foi de $18 \%$ em crianças de 3 a 9 anos de idade. Enquanto no atual estudo, nas EPR e na EPU, 20,77\% e $39,05 \%$, respectivamente responderam que os dentes decíduos anteriores que foram extraídos ou esfoliados apresentavam lesões de cárie.

O compartilhamento de escovas dentárias entre membros de uma mesma família esteve presente entre $3,5 \%$ dos 141 escolares da atual pesquisa. Grigoletto JC, et al. (2006) observaram que 2,7\% dos 336 escolares analisados faziam uso coletivo da escova dental com outros membros da família. Paschoal AD e Rotta J (1992) constataram que $13,42 \%$ dos escolares analisados compartilhavam escova dental com membros da família, por não ter uma escova dental individual.

Apesar da pequena porcentagem de crianças que apresentam esse comportamento, os dados apontados são relevantes e preocupantes, dada a gravidade do problema, visto que o compartilhamento de escova dental pode ser vetor de transmissão de inúmeras doenças infecciosas, virais e parasitárias (GRIGOLETTO JC, et al., 2006). Portanto, a introdução de práticas educativas e acompanhamento destas para modificar a realidade no tocante ao compartilhamento de escovas dentais são de suma importância.

Em ambas as escolas as crianças sentiram dor, embora na pública a ocorrência tenha sido maior (pública 45,31\% privada 25,97\%), com diferença significativa entre elas (Qui2; $p=0,026$ ). As crianças que apresentaram extração indicada, não receberam o atendimento adequado para que essa necessidade fosse atendida, por isso houve a presença da dor, e que pode estar relacionada à falta de acesso ao dentista, como pode ser visto na Figura 4.

Os estudantes da EPU $(67,18 \%)$ frequentam menos o dentista do que os das privadas $(42,85 \%)$, e isto se reflete nos índices ceo-d e CPO-D encontrado nas diferentes escolas, sendo o ceo-d e CPO-D maiores nas EPU (Figuras 2 e 3). No entanto, quando se avalia o ceo-d, se observa que os da EPU apresentam maior índice e, isto está em acordo com os estudos de Hoffmann RH, et al. (2004) e Berti M, et al. (2013) que mostraram resultados semelhantes ao do presente estudo, onde os índices ceo-d e a prevalência de cárie nas crianças de escolas públicas foram maiores do que nas das escolas particulares, porém para 0 índice de CPO-D esses resultados não se assemelham ao do presente estudo.

Ainda no que se refere à cárie dentaria presente nas crianças da EPU e EPR ficou demostrado que a história natural da doença está em desenvolvimento contínuo, como visto nos índices ceo-d e CPO-D. E isso leva a um questionamento: será que a prática do atual modelo de atenção em saúde bucal está sendo eficiente para atender as necessidades atuais que exigem intervenção curativa associada a medidas de prevenção para o enfrentamento da cárie dentária? Carvalho EMOF, et al. (2011) relatam que os cirurgiãesdentistas brasileiros com idade inferior a 40 anos não foram devidamente preparados para atuar na Estratégia Saúde da Família (ESF), que as instituições de graduação em odontologia devem rever a formação para capacitá-los e estimularem o aperfeiçoamento para ações de saúde coletiva.

Por outro lado, os que foram formados antes da ESF, também não tiveram treinamento para executar o processo de trabalho proposto por ela, muitas vezes enxergando apenas o problema, dissociando-o do indivíduo e seu contexto de vida (CARVALHO EMOF, et al., 2011). Assim há a necessidade de capacitação permanente para que os cirurgiões dentistas possam atuar conforme as necessidades de saúde da 
população brasileira. Quando se fala sobre a oferta de cuidados bucais por meio da atenção primaria em saúde, sabe-se que para que tal oferta ocorra na assistência dentro de uma área adscrita (AA), as pessoas precisam estar cadastradas como moradoras na unidade de saúde promotora desta atenção.

No âmbito coletivo, não necessariamente as pessoas são cadastradas; basta que o estabelecimento pertença a AA. Ambas as escolas particulares e pública pertencem à uma mesma AA no município envolvido na pesquisa, assim a mesma UBS presta cuidados coletivos as EPR e EPU. O acolhimento deve oferecer equidade de acesso para todos os usuários, informar as vagas disponíveis e empregar a triagem com o critério de seleção quanto à severidade da doença, e não por determinação de chegada, contribuindo assim para um bom acesso da população ao serviço de saúde (BRASIL, 2008).

Rojas GC (2014) constatou que na prática há falta de acesso aos serviços de saúde bucal no Brasil, destacando o aspecto organizacional, indicando vários fatores como falta de acessibilidade, atraso em obter uma consulta, presença de filas, complexidade para agendamento, falta de insumos, entre outras. A fim de minimizar tais problemas, e pensando na resolutividade das necessidades de saúde bucal das crianças do presente estudo, talvez se pudesse agrupá-las como fazendo parte da AA também para tratamento, isto é, as escolas deveriam estar cadastradas não só para procedimentos coletivos, mas para os procedimentos assistenciais.

Como observado na Figura 4 nas EPU e EPR 15,62\% e 7,79\% respectivamente, (Qui2; $p=0,233$ ) apresentam dificuldade de fala ou interação social, aspectos estes que podem indicar problemas de qualidade de vida quando afetados. Os dentes decíduos são parte da primeira dentição na criança e apesar de eles serem temporários, quando acontece uma destruição severa por cárie e/ou traumatismo e há necessidade de extração, em ambos os casos há perda precoce, gerando impactos negativos na vida da criança (GOLDENFUM GM, 2015). Estas consequências podem gerar a ausência de espaço no arco, evolução de má oclusão, perda de dimensão vertical, dificuldade na formação e erupção do dente permanente sucessor, entre outros.

A nível psicológico/emocional os elementos dentários em condições precárias têm sido o motivo mais frequente do bullying, e isso diminui a autoestima da criança afetando a qualidade de vida e estética (ARTESE F, 2019). No momento em que a criança está no estágio de desenvolvimento, muitas vezes aprendendo a falar, a se comunicar, entrando na escola e convivendo socialmente, esses problemas podem trazer consequências danosas para o desenvolvimento psicossocial da criança.

Os dados do presente estudo no que se referem à cárie de acometimento precoce de dentes decíduos anteriores referindo como esfoliados ou extraídos e a presença de dentes decíduos cariados apresentados no componente cariado do ceo-d, evidenciam que há necessidade de serem desenvolvidas ações de educação em saúde junto às crianças e responsáveis por elas. O Ministério da Saúde do Brasil disponibiliza materiais educativos na seção "Saúde para Você", que inclui crianças e adultos destacando-se a importância da compreensão das orientações, medidas educativas, por meio do Letramento funcional em saúde (PASSAMAI MPB, et al. 2012).

Nas ações de educação em saúde é de suma importância que os conteúdos sejam trabalhados, de forma conjunta entre os profissionais da equipe de saúde, os da educação, as crianças e seus responsáveis a fim de se alcançarem mudanças no quadro epidemiológico de cárie nas crianças do presente estudo. É necessário que os responsáveis compreendam a importância de encaixar na rotina diária o momento para ofertar cuidados bucais aos seus filhos, de modo a thes proporcionar qualidade de vida.

\section{CONCLUSÃO}

A cárie dentária ainda representa um grande problema de saúde pública e fatores socioeconômicos, níveis educacionais e estilo de vida podem estar relacionados com o seu aparecimento. A falta de acesso aos serviços de saúde bucal no Brasil e, muitas vezes, a execução do processo de trabalho adequado na Atenção Básica faz com que a reversão do quadro de cárie de estabelecimento precoce não ocorra e que medidas de educação em saúde contextualizadas não sejam ofertadas. Assim, o presente trabalho evidencia tais questões, concluindo que houve a presença de cárie de estabelecimento precoce nas crianças do estudo e tal presença pode interferir na qualidade de vida dos voluntários. 


\section{REFERÊNCIAS}

1. ARTESE F. The orthodontist's reach in bullying. Dental Press Journal of Orthodontics, $2019 ; 24$ (2): 15-16.

2. BERTI M, et al. Levantamento epidemiológico de cárie dentária em escolares de 5 e 12 anos de idade do município de Cascavel, PR. Cad Saúde Colet, 2013; 21(4): 403-406.

3. BEZERRA ESM, NOGUEIRA AJS. Prevalência de Perdas Dentárias Precoces em Crianças de População Ribeirinha da Região Amazônica. Pesq Bras Odontop Clin Int, 2012; 12(1): 93-98.

4. BRASIL. Ministério da Saúde. Programa Saúde na Escola - PSE. 2007. Disponível em: http://www.planalto.gov.br/ccivil_03/_ato2007-2010/2007/decreto/d6286.htm. Acessado em: 2 de março de 2020.

5. BRASIL. Ministério da Saúde. Secretaria de Atenção à Saúde: Caderno de Atenção Básica, no 17. 1 edição. 2008. Disponível em: https://bvsms.saude.gov.br/bvs/publicacoes/saude_bucal.pdf. Acessado em: 2 de março de 2020.

6. BUSATO AL, Maltz SM. Cariologia: aspectos de dentística restauradora. 1 ed. São Paulo: Artes Médicas; 2014.

7. CARVALHO EMOF, et al. Práticas odontológicas no Programa Saúde da Família: análise crítica. Revista da ABENO, 2011; 10(1): 52-55.

8. COPELANDA WE, et al. Childhood bullying involvement predicts low- grade systemic inflammation into adulthood. Proc Natl Acad Sci, 2014; 111(21): 7570-7575.

9. FOLAYAN MO, ALADE MA. A Compendium on Oral Health of Children around the World: Early Childhood Caries. 1 ed. USA: Nova Science Publishers; 2018.

10. GOLDENFUM GM. Reabilitação estética na cárie precoce da infância: relato de caso. Dissertação (Trabalho de Conclusão de Curso). Universidade Federal do Rio Grande do Sul, Porto Alegre, 2015.

11. GRIGOLETTO JC, et al. Higiene oral e uso compartilhado de escova dental. Rev Odontol UNESP, 2006; 35(2): 175181.

12. HOFFMANN RH, et al. Dental caries experience in children at public and private schools from a city with fluoridated water. Cad Saude Publica, 2004; 20(2): 522-528.

13. MALTZ M, et al.. Cariologia: Conceitos Básicos, Diagnóstico e Tratamento Não Restaurador. 1 ed. São Paulo: Série ABENO Artes Médicas; 2016.

14. MATOS AN. Consequências da perda precoce dos incisivos superiores decíduos e dos molares decíduos sobre o sistema estomatognático. Dissertação (Trabalho de Conclusão de Curso). Universidade Federal de Santa Catarina, Florianópolis, 2002.

15. MCDONALDS RE, AVERY DR, HENNON DK. Odontopediatria. Tratamento dos traumatismos dos dentes e tecidos de suporte. Rio de Janeiro: 1994; 342 p.

16. OWEN DG. The incidence and nature of space closure following the premature extraction of deciduous teeth. A literature survey. Am J Orthod, 1971; 59(1): 37-49.

17. PASCHOAL AD, ROTTA J. Conservação e uso das escovas entre escolares de cinco municípios do ERSA-55 de Casa Branca/SP. Revista Gaúcha de Odontologia, 1992; 40(4): 276-278.

18. PASSAMAI MPB, et al. Letramento funcional em saúde: reflexões e conceitos. Interface Comun Saúde Educ, 2012; 16(4): 301-314.

19. QUDEIMAT MA, FAYLE SA. The longevity of space maintainers: a retrospective study. Pediatr Dent, 1998; 20(4): 267-72.

20. ROJAS GC. Indicadores de saúde bucal em função da organização da demanda: análise baseada no sistema de informação. Dissertação (Mestrado). Universidade Estadual de Campinas, Campinas, 2014.

21. ROSE JS. Early loss of teeth in children. Br Dent J, 1966; 120(6): 275-280.

22. SILVA PDC, et al. Cárie precoce da infância, qualidade de vida e tratamento: revisão de literatura. Revista Uningá Review, 2015; 24(3): 86-89.

23. THOMAZ EBA, et al. Prevalência de protrusão dos incisivos superiores, sobremordida profunda, perda prematura de elementos dentários e apinhamento na dentição decídua. J Bras Odontopediatr Odontol Bebê, 2002; 5(26): $276-282$. 